\title{
Review of: "Texture feature-based machine learning classifier could assist in the diagnosis of COVID-19"
}

\section{Defu Qiu}

1 China University of Mining Technology - Xuzhou

Potential competing interests: The author(s) declared that no potential competing interests exist.

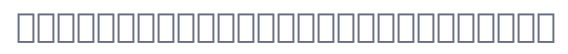

